\title{
Optimization for Locating Emergency Medical Service Facilities: A Case Study for Health Planning from China
}

\author{
Yufan Deng $\mathbb{D}^{1,2}$ \\ Yumeng Zhang ${ }^{1,2}$ \\ Jay Pan $\mathbb{D D}^{1,2}$
}

'HEOA Group, West China School of Public Health and West China Fourth Hospital, Sichuan University, Chengdu, Sichuan, People's Republic of China; ${ }^{2}$ Institute for Healthy Cities and West China Research Center for Rural Health Development, Sichuan University, Chengdu, Sichuan, People's Republic of China
Correspondence: Jay Pan

West China School of Public Health, Sichuan University, No. 17, Section 3, Ren Min Nan Road, Chengdu, 6I004I, Sichuan, People's Republic of China Email panjie.jay@scu.edu.cn
Purpose: Rational location of emergency medical service (EMS) facilities could improve access to EMS, and thus assist in saving patients' lives and improving their health outcomes. A considerable amount of spatial optimization research has been devoted to the development of models to support location planning in the context of EMS, with extensive applications in policy making around the world. However, in China, studies on the location of EMS facilities have not been paid enough attention to, let alone their practical applications. This paper conducted location optimization for EMS facilities in Chengdu, one of the biggest cities in southwest China with more than 16.5 million population, aiming to optimize the EMS system by adding (upgrading) a minimum number of EMS facilities to achieve a given population coverage.

Methods: Location optimization was conducted according to regional health policy goal for the EMS system in Chengdu, China, 2017. The nearest-neighbor approach was used to calculate the shortest travel time based on geographical information system (GIS). The location set covering model was used to formulate the optimization problem under China's context, and genetic algorithm (GA) was employed to determine the optimized locations.

Results: The results showed that a minimum number of 55 new facilities were required to upgrade to EMS facilities to achieve the policy goal of $90 \%$ population coverage of EMS within 15 minutes. Access to EMS also improved substantially in terms of shortest travel time after facility upgrading. The weighted median shortest travel time to EMS facilities in Chengdu decreased by $14.57 \%$, from 6.45 minutes to 5.51 minutes.

Conclusion: Our study showed that the solution could effectively achieve the policy goal of population coverage with a minimum number of new EMS facilities. Our findings would support evidence-based decision-making in future EMS planning in China.

Keywords: emergency medical care, EMS location, genetic algorithm, location set covering problem

\section{Introduction}

Emergency medical service (EMS) is one of the most fundamental services in healthcare system, it is responsible for urgent pre-hospital treatment and patient transfer in the case of medical emergent events. ${ }^{1}$ Timely and efficient provision of EMS has a significant impact on patients' health and recovery, thus plays a vital role in saving patients' lives, improving health outcomes, and reducing rate of mortality. $^{2-4}$ On this account, the improvement of EMS system has long been 
advocated throughout a worldwide range $\mathrm{e}^{5,6}$ and is recognized as an important part of health planning in most countries. $^{7-11}$

Of all the problems arising in EMS planning, locating EMS facilities has gained great attention from both local governments and academia. It has been widely concerned in the city development plan, so as to meet the safety needs of residents and facilitate the realization of health goals. Location problem, which seeks the optimal layout of facilities for a given objective, is one of the most studied area of Operations Research (OR).

A considerable amount of research work has been devoted to developing models to support decisionmakings in the context of facility location. Regardless of details of specific conditions and issues, typical location problems could fall into the following categories according to their optimization objectives: (i) the $p$-median problem; (ii) the $p$-center problem; (iii) the location set covering problem; and (iv) the maximum coverage location problem. ${ }^{12}$ A thorough overview of location science can be found in the book of Laporte et al. ${ }^{13}$ Other popular models and specific topics for location of EMS facilities were carefully reviewed in Aringhieri et $\mathrm{al}^{14}$ and Bélanger et al. ${ }^{15}$

Based on planning objectives, various studies have used different models to assist in locating EMS facilities around the world. Jánošíková et $\mathrm{al}^{16}$ determined the optimized location for current EMS stations in Slovak Republic using $p$-median model, which allowed for a significant improvement in access to EMS without adding new stations. Fritze et $\mathrm{al}^{9}$ restructured the location of EMS stations in Austria by solving maximum coverage location problem. Their solutions realized better coverage with fewer EMS stations than the current situation. Zhu et $\mathrm{al}^{17}$ optimized the location of trauma centers in Shenzhen, China with a modified location set covering model. Results showed that a large service area can be covered and a quick response time can be provided by the optimized solution. Given that important differences can be observed, not only in the ways that EMS are provided ${ }^{18}$ but also in the development level of EMS systems around the world, specific planning objectives could be quite different in policy practice of different countries (and even cities). Therefore, EMS location strategies should be context-specific and support the development of local health system.

This study selected Chengdu as an example to illustrate how OR approach could support in decision-makings in locating EMS facilities in China's context. As one of the biggest cities in southwestern China, Chengdu is currently going through the process of developing into an international metropolis. According to the strategic planning of Chinese government, it has been positioned as one of the national central cities in China. ${ }^{19}$ Serving as a fundamental requirement of residents, building a well-functioned EMS system is clearly an indispensable part of the city's development plans. How to efficiently locate EMS facilities with limited resources has posed great challenge to the local government. Hence, addressing EMS facility location problem from the perspective of OR is urgently needed in order to support policy making on the path towards a better EMS system.

We calculated the shortest travel time from each demand point to EMS facilities using the nearestneighbor method to assess the current access to EMS in Chengdu. According to the city's context of healthcare delivery system and health planning goals, the optimization problem was formulated using the location set covering model. Then, we solved the problem by genetic algorithm (GA). Our study would provide implications on the future EMS planning in China, as well as highlighting the location set covering modelling and GA in regional healthcare resources allocation and healthcare facility location planning, which was rarely adopted in the practices.

\section{Materials and Methods Emergency Medical System in Chengdu, China}

The EMS system in Chengdu was organized at two levels: 1) the command level which was responsible for management services including ambulance dispatching and telephone advice; 2) the service level that provided actual EMS, taking orders from the command level. Facilities in charge of management responsibility included a central emergency command center in downtown Chengdu, and 11 emergency command sub-centers in suburbs. Actual EMS, which included field-first-aids and patient transfer, was implemented by the so-called prehospital emergency care network hospitals (referred as network hospitals below). Network hospitals were evaluated and certified by central emergency command center in Chengdu, following by a regular assessment every 3 years. In consideration of regional differences in distribution of quality medical resources, local governments in periphery 
districts often permitted some other facilities to participate in pre-hospital emergency care. However, these facilities were only able to handle less-emergent cases, uncapable of treating patients in life-threatening conditions.

In 2016, The China's state council issued "Healthy China 2030" as its mid-long term strategic planning in promoting population health. Following it and basing on local socioeconomic development, the city government of Chengdu subsequently announced "Healthy Chengdu 2030". Both of Healthy China and Healthy Chengdu emphasized the EMS system, and regarded improvement of EMS system as one of the main goals in the near future. According to China's National Development and Reform Commission, ${ }^{20}$ the emergency care physicians should be reached by people within 15 minutes. Under the context, we aimed to find the least number of facilities needed to upgrade to network hospitals, in order to achieve a policy goal of $90 \%$ population coverage of EMS within 15 minutes in Chengdu.

\section{Study Area and Data}

As the provincial capital city of Sichuan Province of China, Chengdu is one of the largest and most populated cities in southwestern China with 20 districts. $^{21}$ The total area of the city is about 14.34 thousand $\mathrm{km}^{2}$, with its population reaching 16.04 million in $2017 .^{22}$ As shown in Figure 1A, the population density is relatively high in five downtown districts. The periphery areas are much less populated compared to the central areas.

The data used in this study included supply side, demand side, road network as well as administration boundary data. The supply side data were consisted of three parts: 1) the entry criteria of network hospitals; 2) the list of current network hospitals, and 3) the data of all healthcare facilities of Chengdu in 2017, including facility's name, address, and organization type (hospital, township health center/community health center, and emergency center). All these data were obtained from the Health Bureau of Sichuan Province and the Health Bureau of Chengdu. We then screened the whole database and extracted qualified facilities according to the entry criteria that one facility should meet to become a candidate for network hospital. Briefly speaking, only general hospitals and central township health centers with separate emergency depts were included in our candidate sets. Due to data unavailability, detailed criteria concerning first-aid medicine and medical instruments were not used in this study. After determining the candidate facilities, we used Baidu Map to geocode facility locations according to their names and addresses.
Considering every resident would be regarded as a risk person needing for timely EMS, the number of whole populations was used as estimates of potential demand. ${ }^{23}$ In order to accurately depict the population distribution trend in Chengdu, we applied LandScan $2017,{ }^{24}$ the finest resolution global population gridded data (946 meters $\times 946$ meters) provided by the Oak Ridge National Laboratory, as our demand data. Each grid was treated as a demand point, and grids with zero population were excluded in subsequent analysis.

Road network was used to compute the shortest travel times from each demand point to candidate network hospitals. We gathered road network and administrative boundary data from the 1:250,000-scale Topographic Database of the National Fundamental Geographic Information System of China, which was provided by National Geomatics Center of China (Figure 1B). In applying network analysis, a standard speed limit was assigned to each section of road, based on road class, physical condition, and the Highway Technical Standards of China. Specifically speaking, $120 \mathrm{~km} / \mathrm{h}$, $100 \mathrm{~km} / \mathrm{h}, 80 \mathrm{~km} / \mathrm{h}, 60 \mathrm{~km} / \mathrm{h}$ and $40 \mathrm{~km} / \mathrm{h}$ were set for highways, state roads, provincial roads, county roads and village roads, respectively. For paths where travelling by vehicles was unavailable, the walking speed was set at $5 \mathrm{~km} / \mathrm{h}$.

\section{The Nearest-Neighbor Method}

We used the nearest-neighbor method to calculate travel times from each demand point to candidate network hospitals, and the shortest one was used to assess its current access to EMS. A key assumption of this method was that residents would always choose to go to the nearest facility to seek emergency care, which was in accordance with the utilization of EMS in an ideal world - complying with the principle of proximity, command center would dispatch ambulance from the nearest network hospital as far as possible to reach the patients. Actual situation in a real world could possibly violate this assumption due to the problem of congestion. However, as we adopted this method from a planning perspective, it would be acceptable to make such assumption. Travel times calculated from the nearest-neighbor method would provide a reasonable representation of access to EMS for a given demand point. More specifically, the shortest travel time from each demand point to the nearest network hospital equaled the total travel time spent by vehicles and on walk. Walking speed and speed limits on different road were set as described above, standing for the best potential travel time (minimum response time) in the real world. The nearestneighbor method was performed using OD-matrix and near modules in ArcGIS 10.6. 

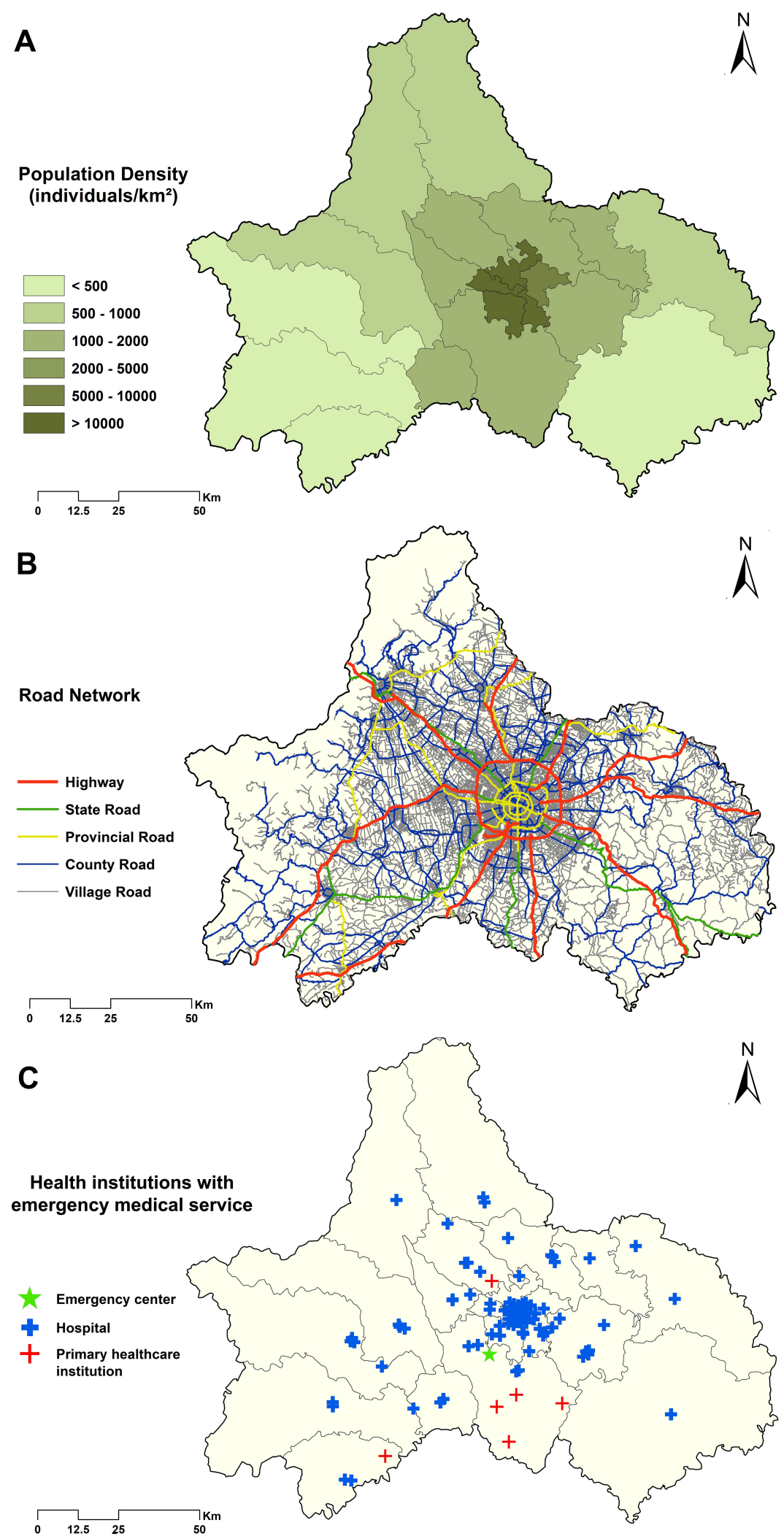

Figure I Population density (A), road network (B) and distribution of network hospitals (C) in Chengdu, 2017. 


\section{Problem Formulation}

To achieve the policy goal of $90 \%$ coverage of EMS within 15 minutes in Chengdu with least number of new network hospitals, the problem of finding optimal facilities to upgrade to network hospitals/to provide EMS fell within the context of location set covering problem. We formulated the location problem as follows:

$$
\begin{gathered}
\min \sum x_{i}, i \in I \\
\text { s.t. } \sum P_{j} y_{j} \geq G, j \in J \\
x_{i} \in\{0,1\}, i \in I \\
y_{j} \in\{0,1\}, j \in J
\end{gathered}
$$

where $I$ and $J$ denoted the set of candidate facilities and the set of uncovered demand points under current EMS system, respectively. $x_{i}$ indicated whether facility $i$ was chosen to upgrade to network hospital (1, if it was chosen; 0 , otherwise) and $y_{j}$ indicated whether demand point $j$ was covered within 15 minutes after facility upgrading $(1$, if it was covered; 0 , otherwise). $P_{j}$ denoted the percentage of population relative to the entire city in each demand point $j . G$ was the gap between current population coverage and predetermined policy goal. To be specific, if the current population coverage was $60 \%, G$ was then set as $30 \%$, since newly upgraded network hospitals were only required to cover $30 \%$ more population, which was currently not covered by existing EMS system. Equation (1) was the main objective function, aiming to minimize the number of new network hospitals needed in order to achieve the policy goal. Equations (2) to (4) were the constraints. Equation (2) explicitly expressed the policy goal-new network hospitals should provide at least $G$ percent coverage to ensure the achievement of $90 \%$ coverage of all population. Equations (3) and (4) were integrality constraints.

It should be noted that different solutions that satisfied the policy goal could be of the same priority, ie, these solutions might require the same number of new network hospitals. In order to simplify the final solution, we modified Equation (1), the objective function, into the following equation:

$$
\min \left(\sum x_{i}-\sum P_{j} y_{j}\right), i \in I
$$

As shown in Equation (5), the additional population coverage brought by new network hospitals was added to the objective function. Noting that population coverage was a percentage ranging from 0 to 1 , by subtracting it from the number of required facilities, we were able to find the solution that realized best coverage among those requiring the same number of new network hospitals. Such modification was simply a computational skill, and should not be confused with multi-objective programming which tried to seek both objectives at the same time.

\section{Solution Methods}

Location set covering problem is a typical NP problem that required solving a large number of polynomials. Metaheuristic methods, such as genetic algorithm, tabu search and ant colony optimization, have gained great popularity in solving this type of problems. ${ }^{25,26}$ In this study, we employed genetic algorithm (GA) to solve the location set covering problem formulated above.

GA was firstly introduced by John Holland based on the concept of evolution theory, analogous to the process of natural selection. ${ }^{27}$ In a typical GA, a population of candidate solutions to an optimization problem was gradually evolved towards better solutions via an iterative process. Specifically, each candidate solution was coded as an individual chromosome with genes, where each gene stood for one candidate facility (ie, equaled the value of $x_{\mathrm{i}}$ ), indicating whether it was chosen or not. The fitness of an individual chromosome was defined using the fitness function. Based on the optimization objective, Equation (5) was initially employed as the fitness function and a smaller value for this function corresponded to a better fitness. To integrate the constraint, we further added a penalty term to the original function - solutions that did not reach the policy goal (Equation (2)) were given a maximal penalty term so that they would have a minimal fitness. The iteration process went: (i) Randomly generating the initial population; (ii) Stochastically selecting individuals with better fitness from the current population to form the new population through a combination of genetic operators, namely mutation and crossover. The best individuals of the current population would also be retained in this step. (iii) Repeating step (ii) until the predetermined number of iterations without fitness improvement has been reached.

Individual with the best fitness in the final population was considered the best solution to the optimization problem. The computational process was conducted using GA package, ${ }^{28,29}$ a package designed for genetic algorithm in R. Parameter settings (Table 1) and function formulation were based on the author's guide. ${ }^{30}$

Weighted median travel time, interquartile range (IQR) and population coverage within 15 minutes were used to compare access to EMS in all districts before and after facility upgrading. Greedy method was used to prioritize 
Table I Parameter Settings in GA

\begin{tabular}{|l|c|}
\hline Parameter & Value \\
\hline Population size & 1000 \\
Number of genes & 463 \\
Probability of crossover & 0.8 \\
Probability of mutation & 0.1 \\
Number of best individuals survived at each & $5 \%$ of population \\
generation & size $(50)$ \\
Number of iterations without improvement & 1500 \\
before stop & \\
\hline
\end{tabular}

the order of facility upgrading in the optimized solution based on the increase of population coverage. All data analyses were conducted in $\mathrm{R} 3.6 .1 .^{31}$

\section{Results}

\section{Current Network Hospitals in Chengdu}

In 2017, there were 95 network hospitals in Chengdu, including 88 hospitals, 6 central township health centers and one emergency center (a special case that located in
CTU, Chengdu Shuangliu International Airport). As shown in Figure 1C, most network hospitals are concentrated in central downtown districts, with fewer facilities distributed in other districts. Even in periphery districts, network hospitals tend to cluster within a small area.

Such pattern of network hospital distribution consequently led to a huge disparity in access to EMS among different districts. Generally, current network hospitals in Chengdu could provide EMS with $78.27 \%$ population covered within 15 minutes (Table 2); thus, newly upgraded network hospitals were only required to cover $11.73 \%$ more population in order to achieve the policy goal. All the populations in five downtown districts have already reached the 15-minute coverage standard (5/20). Besides, four adjacent districts (4/20), including Xindu, Pidu, Longquan and Shuangliu, have also achieved the policy goal of $90 \%$ coverage under current situation. However, the remaining 11 districts $(11 / 20)$ did not reach the policy goal. In the worst two districts, namely Jintang and Jianyang, more than $60 \%$ of the population could not be reached by EMS within 15 minutes.

Table 2 Access to EMS Before and After Facility Upgrading

\begin{tabular}{|c|c|c|c|c|}
\hline & \multicolumn{2}{|c|}{ Current Status } & \multicolumn{2}{|c|}{ After Facility Upgrading } \\
\hline & $\begin{array}{l}\text { Median Shortest Travel Time } \\
\text { (IQR) }\end{array}$ & $\begin{array}{l}\text { Population } \\
\text { Coverage } \\
\text { in } 15 \text { Min }\end{array}$ & $\begin{array}{l}\text { Median Shortest Travel Time } \\
\text { (IQR) }\end{array}$ & $\begin{array}{l}\text { Population Coverage in } 15 \\
\text { Min }\end{array}$ \\
\hline Total & $6.45(10.65)$ & $78.27 \%$ & 5.51 (7.17) & $90.05 \%$ \\
\hline District & & & & \\
\hline Chenghua & $2.55(1.82)$ & $100.00 \%$ & $2.55(1.82)$ & $100.00 \%$ \\
\hline Jinniu & $3.26(3.39)$ & $100.00 \%$ & $3.26(3.39)$ & $100.00 \%$ \\
\hline Jinjiang & $2.26(2.27)$ & $100.00 \%$ & $2.26(2.27)$ & $100.00 \%$ \\
\hline Qingyang & $2.10(1.47)$ & $100.00 \%$ & $2.10(1.47)$ & $100.00 \%$ \\
\hline Wuhou & $2.46(1.78)$ & $100.00 \%$ & $2.46(1.78)$ & $100.00 \%$ \\
\hline Pidu & $6.65(5.87)$ & $96.80 \%$ & $6.28(4.74)$ & $99.33 \%$ \\
\hline Xindu & $7.90(5.96)$ & $94.86 \%$ & $7.78(5.5 \mathrm{I})$ & $97.81 \%$ \\
\hline Longquan & $5.74(6.18)$ & $93.18 \%$ & $4.95(5.50)$ & $96.21 \%$ \\
\hline Shuangliu & $7.47(5.85)$ & $92.63 \%$ & $6.93(5.74)$ & $95.06 \%$ \\
\hline Xinjin & $10.37(5.48)$ & $85.46 \%$ & $10.28(5.09)$ & $88.05 \%$ \\
\hline Wenjiang & $8.82(7.64)$ & $82.95 \%$ & $7.29(4.29)$ & $98.83 \%$ \\
\hline Qingbaijiang & $10.42(6.40)$ & $76.85 \%$ & $8.02(5.31)$ & $92.39 \%$ \\
\hline Dujiangyan & $10.81(14.27)$ & $64.46 \%$ & $8.27(5.68)$ & $92.00 \%$ \\
\hline Chongzhou & I2.57 (9.59) & $61.17 \%$ & $8.90(7.26)$ & $88.01 \%$ \\
\hline Pujiang & I 3.69 (I I.87) & $58.34 \%$ & $10.19(7.91)$ & $80.96 \%$ \\
\hline Dayi & 13.78 (9.88) & $56.39 \%$ & $10.12(6.83)$ & $81.85 \%$ \\
\hline Pengzhou & I 3.87 (10.54) & $55.34 \%$ & $8.17(6.45)$ & $88.59 \%$ \\
\hline Qionglai & I5.85 (I8.45) & $47.34 \%$ & $10.30(11.43)$ & $70.09 \%$ \\
\hline Jintang & $18.90(16.30)$ & $37.26 \%$ & I I.32 (9.78) & $70.21 \%$ \\
\hline Jianyang & $34.35(20.48)$ & $10.94 \%$ & $15.93(14.37)$ & $46.73 \%$ \\
\hline
\end{tabular}

Abbreviation: IQR, interquartile range. 
As listed in Table 2, a wide disparity also existed in the weighted median shortest travel time among districts. The weighted median shortest travel time in Chengdu is 6.45 minutes, with subordinate districts ranging from 2.10 minutes in Qingyang to $34.35 \mathrm{~min}$ utes in Jianyang. Figure 2A showed the visualization of shortest travel time in each demand point. The bottom two districts with regard to population coverage, namely Jianyang and Jintang, were both located in eastern corner of Chengdu. Due to the lack of network hospitals, their shortage of timely EMS was explicitly shown in the figure, with large areas covered by red colors.

\section{Optimized Network Hospitals in Chengdu}

After data screening, 463 facilities which met the basic entry criteria of network hospitals were set as our candidate set. The computational result showed that Chengdu could reach a $90 \%$ coverage of all population in 15 minutes, assisted by a minimum number of 55 new network hospitals. The locations and upgrading priority of these newly upgraded facilities were shown in Figure 3. Detailed results of greedy methods were listed in Table S1. In general, all the new network hospitals were sparsely distributed outside the downtown districts, with more facilities located in districts with worse access to EMS. As for the facility type, 10

A

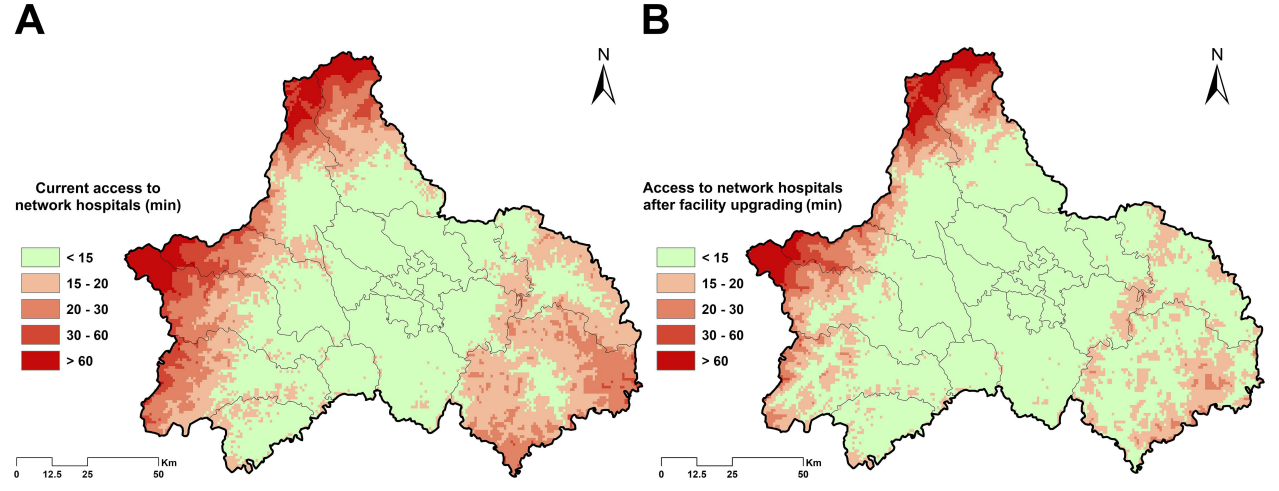

Figure 2 Access (shortest travel time) to network hospitals before (A) and after facility upgrading (B).

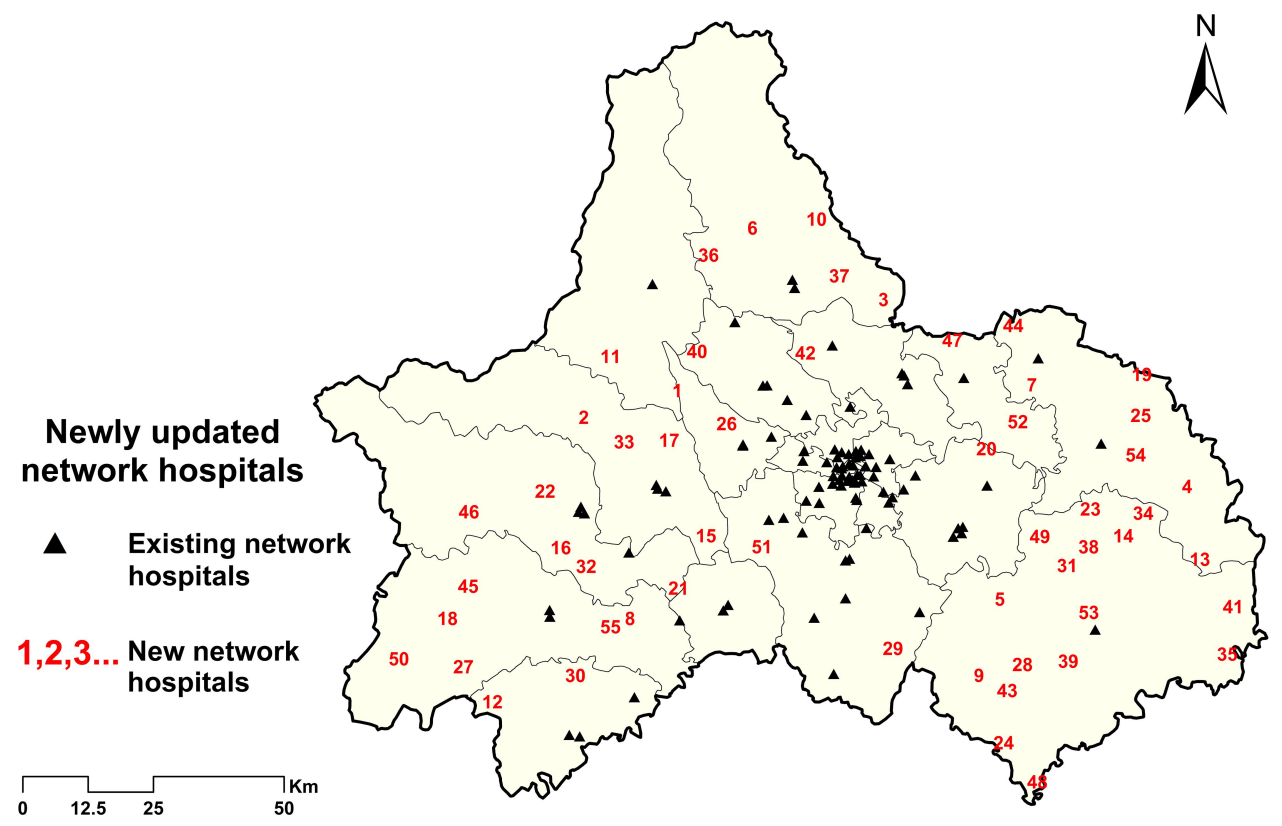

Figure 3 Distribution of current and newly upgraded network hospitals in Chengdu. Numbers denoted the upgrading order of each facility. 
of them were hospitals, the remaining 45 were all township health centers.

As shown in Figure $2 \mathrm{~B}$ and Table 2, after facility upgrading, population coverage within 15 minutes in Chengdu could reach $90.05 \%$. The weighted median shortest travel time decreased by $14.57 \%$, from 6.45 minutes to 5.51 minutes. The IQR also decreased from 10.65 minutes to 7.17 minutes. Three more districts, including Wenjiang, Qingbaijiang and Dujiangyan, achieved the 90\% coverage goal when calculating the coverage index at district-level (12/20). The next three districts, namely Pengzhou, Xinjin and Chongzhou, all drew close to the policy goal, with district coverage exceeding $88 \%$.

Although still showing a relatively lower converge rate, the bottom two districts both improved greatly in access to EMS. The weighted median shortest travel time in Jintang decreased by $40.11 \%$, from 18.90 minutes to 11.32 minutes, with $70.21 \%$ population (vs $37.26 \%$ before) covered by EMS within 15 minutes. Jianyang improved the most with the help of newly selected network hospitals - the shortest travel time was less than half that of the current situation, and population coverage within 15 minutes raised from $10.94 \%$ to $46.73 \%$, increasing by more than $300 \%$.

\section{Sensitivity Analyses}

In order to explore potential solutions under different conditions from the planning perspective, we further modified our model to conduct three sensitivity analyses: 1) reselecting all network hospitals from candidate facilities, which implied that existing network hospitals might not be included in the EMS system in final solution; 2) relaxing entry criteria for candidate facilities - we included all hospitals, whether qualified or not, plus qualified central township health centers in the candidate set; 3) changing the standard of emergency response time from 15 to 12 minutes in the policy goal.

We provided three sensitivity analyses as complements to the main result, brief results were listed in Table 3 .

The first analysis addressed the same problem as the main research from the perspective of facility relocation. All qualified facilities that met the entry criteria, including existing network hospitals, were set as candidate facilities. Therefore, this model aimed to reselect all network hospitals to find the most resource saving solution. Eighty-one facilities were finally selected and consisted of a new EMS system that covered $90.01 \%$ population within 15 minutes. It was worth noting that the number of network hospitals required was even less than that of the current EMS system (95 network hospitals).

The second analysis relaxed the entry criteria of network hospitals. In addition to the existing 463 candidates, 290 other hospitals were included in the candidate set. The solution to this modified model showed little difference from the main results: 54 facilities were upgraded to network hospitals, leading to a $90.13 \%$ coverage in Chengdu.

The third analysis reduced the emergency response time from 15 to 12 minutes. After appropriate calculation, we found that even if all qualified facilities were set as network hospitals, it was still impossible to achieve the policy goal of $90 \%$ coverage (these facilities could only reach a maximum of $89.23 \%$ coverage). In this regard, all 1173 healthcare facilities in Chengdu were included in candidate set, and the same reselecting strategy was applied as in the first analysis. Results showed that with a minimum number of 206 facilities, the coverage rate within 12 minutes could reach $90.02 \%$ in Chengdu.

\section{Discussion}

The time needed to reach EMS was considered a key performance indicator of EMS system. Timely access to

Table 3 Summary Results of Three Sensitivity Analyses

\begin{tabular}{|c|c|c|c|}
\hline Objective & Strategy & Candidate Set & Result \\
\hline \multirow{2}{*}{$\begin{array}{l}\text { Objective I: Achieving } 90 \% \\
\text { coverage of EMS within } 15 \text { min }\end{array}$} & \multirow{2}{*}{$\begin{array}{l}\text { Reselecting all } \\
\text { network hospitals }\end{array}$} & Qualified hospitals and township health centers & 8I Facilities were selected \\
\hline & & 558 facilities in total & $90.01 \%$ coverage within $15 \mathrm{~min}$ \\
\hline \multirow{2}{*}{$\begin{array}{l}\text { Objective 2: Achieving } 90 \% \\
\text { coverage of EMS within } 15 \text { min }\end{array}$} & \multirow{2}{*}{$\begin{array}{l}\text { Upgrading to } \\
\text { network hospitals }\end{array}$} & Hospitals plus qualified township health centers & 54 New facilities were upgraded \\
\hline & & 753 facilities in total & $90.13 \%$ coverage within $15 \mathrm{~min}$ \\
\hline \multirow{2}{*}{$\begin{array}{l}\text { Objective 3: Achieving } 90 \% \\
\text { coverage of EMS within } 12 \mathrm{~min}\end{array}$} & \multirow{2}{*}{$\begin{array}{l}\text { Reselecting all } \\
\text { network hospitals }\end{array}$} & All healthcare facilities in Chengdu & 206 Facilities were selected \\
\hline & & II73 facilities in total & $90.02 \%$ coverage within $12 \mathrm{~min}$ \\
\hline
\end{tabular}


EMS could substantially reduce the rate of mortality and improve overall health outcomes. For this reason, emergency response time has gained great popularity in health planning around the world. For example, Italian law has made it mandatory for EMS facilities to respond to urgent call within 8 minutes in urban area. ${ }^{14}$ Local governments in lower Austria required that $95 \%$ of population should be reachable by emergency care physicians within 20 minutes. In China, EMS systems in many provinces have been developed with the goal of constructing a "15-minute emergency response circle" or setting up a "3-5 km service radius" for EMS facilities. After the announcement of "Healthy China 2030", 32 Chinese government was actively promoting both capacity and efficiency of EMS system across the country. However, compared with the advanced development in location optimization techniques, regional policy making in China was relatively lagging with little or no use of these scientific methods, which often resulted in irrational locations of EMS facilities under resource constraint. Therefore, locating EMS facilities using operation research method was expected to uncover latent defects in current EMS system and assist in decisionmakings in future EMS planning. Our study also highlighted the necessity of modernization of regional health planning, which would be of great advantage in real practice.

Based on current network hospitals, our study found a significant disparity in access to EMS across different districts in Chengdu. Five downtown districts were reported to have a full population coverage of EMS within 15 minutes, while periphery districts like Jianyang and Jintang still suffering from a shortage of EMS, with coverage rate less than $40 \%$ under current situation. Our result was consistent with previous studies focusing on access to healthcare resources in Sichuan province. ${ }^{33,34}$ Since health resource supply was considered one of the most important contributors to residents' access to health care, ${ }^{35}$ clustered distribution of network hospitals would certainly widen the existing regional gaps in access to EMS, and further induce disparities in healthcare outcomes.

Applying location set covering modelling and solving it by GA, we provided an optimized solution in selecting newly upgraded network hospitals in Chengdu. Our solution could reach the policy goal of $90 \%$ population coverage within 15 minutes, with a minimum number of 55 new network hospitals. The optimized location of EMS facilities also promoted access to EMS in Chengdu, with weighted median shortest travel time decreased by $14.57 \%$. Results suggested that significant improvements were achieved in district level as well. After facility upgrading, more than $50 \%$ of the districts $(12 / 20)$ achieved the policy goal of $90 \%$ coverage. Currently, location optimization was rarely adopted in regional practices of healthcare facility location. Above results validated the effectiveness of our optimization model, and demonstrated that location optimization could play an important role in future EMS planning in China.

It should be noted that in the optimized solution, 45 of 55 $(83.33 \%)$ newly upgraded network hospitals were all township health centers. According to the Chinese Ministry of Health, township health center was officially classified as primary healthcare institution in China. ${ }^{33,36}$ However, in addition to primary care services, they also took charge of medical care services in areas with scarce healthcare resources. The solution of the location optimization model has pointed out the vital role of township health centers in improving access to EMS in rural areas. As parts of the primary healthcare system, township health centers were more evenly distributed compared to higher-level hospitals. Such pattern of distribution was consistent with the universal coverage goal of EMS, and therefore township health centers would be ideal candidates for future network hospitals in rural areas. One of the obstacles that hindered the participation of township health centers in delivering EMS was their lower level of service capability and quality. Even if the entry criteria set for township health centers was relatively looser compared to that of hospitals, satisfying all the requirements was still a great challenge for most township health centers in rural areas. Pooling regional resources on a couple of township health centers to construct regional medical centers would be a possible solution. By doing so, certain township health centers would be able to develop at a rapid pace, substantially improving their service capability so as to meet the entry criteria of network hospital. In addition, training programs concerning emergency medical care were highly encouraged as an important approach to improve EMS ability in rural areas.

The clustered distribution of existing network hospitals also revealed a potential defect of current EMS system in Chengdu. The qualification assessment for network hospital was passively conducted by central emergency command center after applications of willing facilities. Considering the clustered distribution tendency of quality healthcare resources, the probability was relatively high that a fair proportion of network hospitals would concentrate within small areas. Such distribution pattern would probably result in resource maldistribution problem which usually led to a waste of resources, thus harm the efficiency of overall 
EMS system. As shown by the first sensitivity analysis, a minimum number of 81 network hospitals were already sufficient to achieve the same policy goal of $90 \%$ coverage within 15 minutes. Although 81 facilities were not enough in real-world situations, the gap between solutions (81 vs 150) was large enough to uncover the latent maldistribution problem in the current EMS system. Similar result was also obtained in Austria by Fritze et al $^{9}$. Various measures could be adopted in order to alleviate such problem. On the one hand, the guidance function of government should be brought into full play to encourage wide participation of healthcare facilities in providing EMS. On the other hand, for related policymakers and planners, rational location strategies should always be kept in mind to avoid inefficient location of EMS facilities. Government officials were encouraged to seek professional help from experts of management science or Operational Research.

We recognized that this study had several limitations. First, we used shortest travel time as a representative of potential emergency response time and calculated this index in the best possible situation. However, actual emergency response in the real world would cost much more time in consideration of potential traffic jams and extra preparation time before the departure of ambulance. Future studies could make use of more detailed data to find solutions under real-world conditions. Second, due to data unavailability, we could not obtain the list of healthcare facilities in adjacent cities of Chengdu. Problem of cross-regional medical practice was inevitable in this study. Residents living close to the administrative boundary of Chengdu might seek EMS from adjacent cities, thus the actual access to EMS would be better for these people comparing to our results. However, the frontier region of Chengdu were mostly rural areas with deficient healthcare resource. Therefore, cross-regional medical practice would have a minor influence on our study results. Third, our study dealt with a practical issue using standard operation research methods that might limit our research depth. With new techniques and detailed data, it would be possible to perform more in-depth optimization and provide more comprehensive solutions in the future.

\section{Conclusion}

Improving efficiency of EMS system has long been called for in different countries around the world. In this paper, we addressed the location set covering problem using genetic algorithm in order to find the least number of facilities required to achieve the policy goal of $90 \%$ coverage of EMS within 15 minutes in Chengdu. Optimization model showed that only a small number of newly upgraded network hospitals would be sufficient to achieve the policy goal. Our findings will benefit policymakers and planners in future EMS planning in Chengdu, and highlight the importance of location optimization in health facility location planning, which was rarely adopted in policy making in China.

\section{Abbreviations}

EMS, emergency medical service; GIS, geographical information system; GA, genetic algorithm; IQR, interquartile range.

\section{Data Sharing Statement}

The LandScan gridded population dataset used in the current study is available in the Oak Ridge National Laboratory's repository: https://landscan.ornl.gov/. The facility data of Chengdu that support the findings of this study are available from the Health Bureau of Sichuan Province and the Health Bureau of Chengdu, but restrictions apply to the availability of these data, which were used under license for the current study, and so are not publicly available. Data are however available from the authors upon reasonable request and with permission of the local Health Bureaus.

\section{Ethics Approval and Informed Consent}

This study was exempted for ethics approval that we only used aggregated population data from a publicly available repository (LandScan gridded population dataset) in our search. As for the facility data, we have obtained permissions from the Health Bureau of Sichuan Province and the Health Bureau of Chengdu to use the information for our research purpose.

\section{Acknowledgments}

We would thank Xiuli Wang and Xiaoshuang Zhao from Sichuan University for their research assistance and valuable suggestions.

\section{Author Contributions}

All authors made substantial contributions to conception and design, acquisition of data, or analysis and interpretation of data; took part in drafting the article or revising it critically for important intellectual content; agreed to submit to the current journal; gave final approval of the version to be published; and agree to be accountable for all aspects of the work. 


\section{Funding}

Financial support for this study was provided by the National Natural Science Foundation of China (Grant No. 71874116 and 72074163), Ministry of Education of China (Grant No. 18YJA790062 and 20YJC790179), Chengdu Federation of Social Science Association (Grant No. ZZ05), Sichuan University (Grant No. 2018hhf-27 and SKSYL201811), China Postdoctoral Science Foundation (Grant No. 2020M673274), Science and Technology Department of Sichuan Province (Grant No. 2020JDR0151) and China Medical Board (Grant No. 17-276). The funding agreement ensured the authors' independence in designing the study, interpreting the data, writing, and publishing the report.

\section{Disclosure}

The authors declare that there are no conflicts of interest.

\section{References}

1. Calvello EJB, Broccoli M, Risko N, et al. Emergency care and health systems: consensus-based recommendations and future research priorities. Acad Emergency Med. 2013;20(12):1278-1288. doi:10.1111/acem.12266

2. Bhutta ZA, Das JK, Bahl R, et al. Can available interventions end preventable deaths in mothers, newborn babies, and stillbirths, and at what cost? Lancet. 2014;384(9940):347-370. doi:10.1016/S0140-6736(14)60792-3

3. Gauss T, Ageron F-X, Devaud M-L, et al. Association of prehospital time to in-hospital trauma mortality in a physician-staffed emergency medicine system. JAMA Surg. 2019;154(12):1117-1124. doi:10.1001/jamasurg.2019.3475

4. Tansley G, Schuurman N, Bowes M, et al. Effect of predicted travel time to trauma care on mortality in major trauma patients in Nova Scotia. Can J Surg. 2019;62(2):123-130. doi:10.1503/cjs.004218

5. World Health Assembly. Health systems: emergency-care systems [WHA Resolution 60.22]; 2007.

6. Meara JG, Leather AJM, Hagander L, et al. Global Surgery 2030: evidence and solutions for achieving health, welfare, and economic development. Lancet. 2015;386(9993):569-624. doi:10.1016/S0140-6736(15)60160-X

7. Başar A, Çatay B, Ünlüyurt T. A multi-period double coverage approach for locating the emergency medical service stations in Istanbul. $J$ Oper Res Soc. 2017;62(4):627-637. doi:10.1057/jors.2010.5

8. Andersson H, Granberg TA, Christiansen M, Aartun ES, Leknes H. Using optimization to provide decision support for strategic emergency medical service planning - Three case studies. Int $J$ Med Inform. 2020;133:103975. doi:10.1016/j.ijmedinf.2019.103975

9. Fritze R, Graser A, Sinnl M. Combining spatial information and optimization for locating emergency medical service stations: a case study for Lower Austria. Int $J$ Med Inform. 2018;111:24-36. doi:10.1016/j.ijmedinf.2017.12.008

10. Liu M, Yang D, Hao F. Optimization for the locations of ambulances under two-stage life rescue in the emergency medical service: a Case Study in Shanghai, China. Math Probl Eng. 2017;2017:1-14. doi: $10.1155 / 2017 / 1830480$

11. Moeini M, Jemai Z, Sahin E. Location and relocation problems in the context of the emergency medical service systems: a case study. Central Eur J Oper Res. 2014;23(3):641-658. doi:10.1007/s10100-014-0374-3

12. Ahmadi-Javid A, Seyedi P, Syam SS. A survey of healthcare facility location. Comput Oper Res. 2017;79:223-263. doi:10.1016/j. cor.2016.05.018
13. Laporte G, Nickel S, Saldanha da Gama F. Location Science. Springer International Publishing; 2015.

14. Aringhieri R, Carello G, Morale D. Supporting decision making to improve the performance of an Italian Emergency Medical Service. Ann Oper Res. 2016;236(1):131-148. doi:10.1007/s10479-013-1487-0

15. Bélanger V, Ruiz A, Soriano P. Recent optimization models and trends in location, relocation, and dispatching of emergency medical vehicles. Eur J Oper Res. 2019;272(1):1-23. doi:10.1016/j.ejor.2018.02.055

16. Jánošíková L', Kvet M, Jankovič $\mathrm{P}$, Gábrišová L. An optimization and simulation approach to emergency stations relocation. Central Eur J Oper Res. 2019;27(3):737-758. doi:10.1007/s10100-019-00612-5

17. Zhu Y, Du Q, Tian F, Ren F, Liang S, Chen Y. Location optimization using a hierarchical location-allocation model for trauma centers in Shenzhen, China. ISPRS Int J Geo-Inf. 2016;5(10):190. doi:10.3390/ijgi5100190

18. Dick, Wolfgang F. Anglo-American vs. Franco-German emergency medical services system. Prehosp Disaster Med. 2003;18(01):29-37. doi:10.1017/S1049023X00000650

19. State Council of the People's Republic of China. Chengdu Chongqing Urban Agglomeration planning; 2016.

20. National Development and Reform Commission. Construction planning of EMS system for public health emergency; 2003.

21. Ministry of Civil Affairs. Statistical report of administrative divisions in China; 2017.

22. Health Committee of Sichuan Province. Sichuan health statistical yearbook 2018; 2018.

23. Li X, Wang F, Yi H. A two-step approach to planning new facilities towards equal accessibility. Environ Planning B Urban Anal City Sci. 2016;44(6):994-1011. doi:10.1177/0265813516657083

24. Budhendra B, Edward. LandScan USA: a high-resolution geospatial and temporal modeling approach for population distribution and dynamics. Geojournal. 2007. doi:10.1007/s10708-007-9105-9

25. Blum C. Ant colony optimization: introduction and recent trends. Phys Life Rev. 2005;2(4):353-373. doi:10.1016/j.plrev.2005.10.001

26. Glover F, Taillard E. A user's guide to tabu search. Ann Oper Res. 1993;41(1):1-28. doi:10.1007/BF02078647

27. Holland J. Adaptation in Natural and Artificial Systems: An Introductory Analysis with Application to Biology. University of Michigan Press; 1975.

28. Scrucca L. GA: a Package for Genetic Algorithms in R. J Stat Softw. 2013;53(4):37. doi:10.18637/jss.v053.i04

29. Scrucca L. On some extensions to GA package: hybrid optimisation, parallelisation and islands evolution. $R J .2017 ; 9(1): 20$. doi:10.32614/RJ2017-008

30. Scrucca L. A quick tour of GA; 2019. Available from: https://cran. r-project.org/web/packages/GA/vignettes/GA.html\#references. Accessed April 8, 2021.

31. $\mathrm{R}$ Core Team. R: a language and environment for statistical computing. R Foundation for Statistical Computing; 2020. Available from: https://www.R-project.org/. Accessed April 8, 2021.

32. Tan X, Liu X, Shao H. Healthy China 2030: a Vision for Health Care. Value Health Reg Issues. 2017;12:112. doi:10.1016/j.vhri.2017.04.001

33. Wang X, Yang H, Duan Z, Pan J. Spatial accessibility of primary health care in China: a case study in Sichuan Province. Soc Sci Med. 2018;209:14-24. doi:10.1016/j.socscimed.2018.05.023

34. Pan J, Liu H, Wang X, Xie H, Delamater PL. Assessing the spatial accessibility of hospital care in Sichuan Province, China. Geospat Health. 2015;10(2):384. doi:10.4081/gh.2015.384

35. Mathison DJ, Chamberlain JM, Cowan NM, et al. Primary care spatial density and nonurgent emergency department utilization: a new methodology for evaluating access to care. Acad Pediatr. 2013;13(3):278-285. doi:10.1016/j.acap.2013.02.006

36. Yao Q, Liu C, Ferrier J, Liu Z, Sun J. Urban-rural inequality regarding drug prescriptions in primary care facilities - A pre-post comparison of the National Essential Medicines Scheme of China. Int J Equity Health. 2015;14:58. doi:10.1186/s12939-015-0186-7 


\section{Publish your work in this journal}

Risk Management and Healthcare Policy is an international, peerreviewed, open access journal focusing on all aspects of public health, policy, and preventative measures to promote good health and improve morbidity and mortality in the population. The journal welcomes submitted papers covering original research, basic science, clinical \& epidemiological studies, reviews and evaluations,

guidelines, expert opinion and commentary, case reports and extended reports. The manuscript management system is completely online and includes a very quick and fair peer-review system, which is all easy to use. Visit http://www.dovepress.com/testimonials.php to read real quotes from published authors. 